\title{
Piperine alleviates osteoclast formation through the p38/c-Fos/NFATc1 signaling axis
}

\author{
Vishwa Deepak $^{1}$, Marlena C. Kruger ${ }^{2,3,4}$, Annie Joubert ${ }^{1}$, Magdalena Coetzee ${ }^{1,4^{*}}$ \\ ${ }^{1}$ Department of Physiology, Faculty of Health Sciences, University of Pretoria, Pretoria, South Africa \\ ${ }^{2}$ School of Food and Nutrition, Massey Institute of Food Science and Technology, Massey University, \\ Palmerston North, New Zealand \\ ${ }^{3}$ Department of Human Nutrition, Faculty of Health Sciences, University of Pretoria, Pretoria, South Africa \\ ${ }^{4}$ Associate of the Institute for Food, Nutrition and Well-being, University of Pretoria, Pretoria, South Africa
}

*Author to whom correspondence should be addressed:

Dr. Magdalena Coetzee, Department of Physiology, University of Pretoria, Private Bag X323, Arcadia 0007, Pretoria, South Africa

E-Mail: magdalena.coetzee@ up.ac.za; Tel.: +27-12-319-2445; Fax: +27-12-321-1679 


\begin{abstract}
Increased bone fracture is one of the health risk factors in patients with bone loss related disorders such as osteoporosis and breast cancer metastasis to bone. Over activity of osteoclasts leads to uncoupling of bone remodeling favoring bone loss over bone formation. Receptor activator of nuclear factor- $\kappa \beta$ ligand (RANKL) triggers the differentiation pathway leading to multinucleated osteoclast formation. Modulation of RANKL or its downstream signaling pathways involved in osteoclast formation is of significant interest in the development of anti-resorptive agents. In this study, we investigated the effects of piperine, an alkaloid present in Piper nigrum L. on osteoclast formation. Piperine inhibited tartrate-resistant acid phosphatase (TRAP)-positive multinucleated osteoclast formation in murine RAW264.7 macrophages and human CD14+ monocytes induced by RANKL and breast cancer cells. Piperine attenuated the p38-mitogen activated protein kinase (MAPK) pathway activation, while the extracellular-signal-regulated kinase (ERK), c-Jun N-terminal kinase (JNK) or NF- $\beta \beta$ pathways downstream of RANKL remained unaffected. Concomitantly, expression of c-Fos and nuclear factor of activated T-cells, cytoplasmic 1 (NFATc1), the key transcription factors involved in osteoclastogenesis were remarkably inhibited by piperine. Furthermore, piperine disrupted the actin ring structure and bone resorption, a characteristic hallmark of osteoclasts. Collectively, these results suggest that piperine inhibits osteoclast differentiation by suppressing the $\mathrm{p} 38 / \mathrm{NFATc1/c-Fos}$ signaling axis.
\end{abstract}

Keywords: RANKL, osteoclast, breast cancer, bone, RAW264.7, CD14+ monocytes 


\section{Introduction}

Osteoclasts are specialized bone resorbing multinucleated cells derived from hematopoietic cells of the monocyte/macrophage lineage (1). The receptor activator of nuclear factor kappa- $\beta$ (RANK) and its ligand (RANKL) are key molecules involved in differentiation and activation of osteoclasts from precursor cells (2). On the other hand, osteoblasts arising from progenitor cells of the mesenchymal lineage are responsible for bone formation and mineral deposition (3). The interplay among osteoblasts and osteoclasts are coupled in a manner that the processes of bone formation and bone resorption termed bone remodeling does not supersede each other. Intriguingly, bone remodeling is often aggravated during certain diseases such as osteoporosis, cancer metastasis to bone, rheumatoid and osteo-arthritis $(4,5)$. Excessive bone loss as seen in aforementioned pathological conditions correlate either with elevated osteoclast formation or augmented osteoclast activity. In line with this, ageing also constitutes to the negative effects seen in bone-loss related diseases as stemness and differentiation potential of mesenchymal progenitors towards osteoblast lineage is afflicted $(6,7)$.

RANKL and its receptor RANK are key factors involved in the initiation of osteoclast formation (8). RANKL, a member of the tumor necrosis factor (TNF) family triggers the formation of osteoclasts and plays an important role in their survival (8). RANKL is produced in membrane-bound form by cells of osteoblast lineage and activated T-cells (9). Both, the soluble as well as membrane bound form of RANKL are produced by activated T-cells and certain type of cancer cells (prostate- and breast cancer), although production of soluble RANKL indicates pathological conditions (9-11).

In the bone microenvironment, physical interaction between osteoblasts and osteoclast precursors activates RANK resulting in initiation of the downstream signaling cascade involved in osteoclast formation. During rheumatoid arthritis, production of soluble RANKL by activated T-lymphocytes can also trigger osteoclastogenesis from osteoclast precursors causing joint destruction (12). Osteoblasts also produce and secrete a decoy receptor of RANK, osteoprotegerin (OPG) (2). The decoy receptor binds to all forms of RANKL and neutralizes its functions. The RANKL/RANK/OPG system is instrumental in bone remodeling.

Macrophage-colony stimulating factor (M-CSF) is a cytokine produced by various cells including osteoblasts and acts in a paracrine manner to maintain the survival and proliferation of osteoclast progenitors (13). Trimerization of RANK occurs when RANKL binds to the receptor leading a subsequent step that involves binding to an adaptor molecule, TNF receptor-associated factor 6 (TRAF6) (14). This activates a sequential chain of events comprising activation of IкB $\alpha / \beta$ kinase (IKK), NF- $\kappa \beta$, MAPKs, c-Fos and NFATc1 resulting in osteoclast formation.

Since, RANKL-induced signaling pathway is critical for osteoclast formation, attenuation of RANKL or effectors downstream of RANKL is a promising therapeutic option against osteoclastogenesis (15). As reported by Newman et al, approximately $48.6 \%$ of currently approved drugs by FDA are either natural products or are directly derived from them and thereby serve as an essential source of lead compounds (16). Piperine is one of the major constituents of black pepper, Piper nigrum Linn. and long pepper, Piper longum Linn. Piper species have been used as an oriental medicinal plant for curing a wide range of diseases such as diarrhoea, gastric ailments and cholera in Chinese-, Indian- and pacific islands traditional medicines (17). Piperine is a major bioactive compound reported to possess anti-cancer-, analgesic, anti-inflammatory- and anti-oxidant properties and is also used to increase bioavailability of drugs and nutrients (18-20). Oral supplementation of piperine has been reported to 
suppress lung carcinogenesis in mice (21). Vijayakumar et al. observed that piperine can reduce high-fat diet induced oxidative stress (22). Piperine has been shown to increase plasma levels of orally supplemented coenzyme $\mathrm{Q}_{10}$ (23). Moreover, piperine has also been demonstrated to inhibit cancer stem cell self-renewal properties (24). Park et al. have demonstrated antiadipogenic properties of piperine in 3T3-L1 cells in vitro (25). Although it is widely used and has an extensive range of pharmacological activities, little is known about the effects of piperine on osteoclastogenesis. Hence, we sought to analyze the potential effects of piperine on osteoclast formation. We provide first line of evidence that piperine has potential anti-osteoclastogenic effects by disruption of the p38/cFos/NFATc1 signaling axis and suppression of osteoclast function by disruption of cytoskeletal rearrangement.

\section{Methods}

\subsection{Reagents}

Dulbecco's Modified Eagle Medium (DMEM), $\alpha$-MEM and heat-inactivated fetal bovine serum (FBS) were obtained from GIBCO (Grand Island, NY). Antibiotic-antimycotic solution containing $100 \mathrm{U} / \mathrm{ml}$, penicillin, $100 \mathrm{~g} / \mathrm{ml}$ streptomycin, and $0.25 \mathrm{~g} / \mathrm{ml}$ fungizone was supplied by Highveld Biological (Johannesburg, South Africa). Piperine, phalloidin-Atto-488, TRAP kit and all other chemicals of research grade were obtained from Sigma-Aldrich Inc. (St Louis, MO). Human M-CSF was supplied by Insight Biotechnology (Middlesex, UK). Mouse- and human RANKL were acquired from R\&D Systems (Minneapolis, MN) respectively. All components for the magnetic separation of CD14+ monocytes were supplied by Miltenyi Biotec (San Diego, CA). Alamar blue reagent, cell extraction buffer, NBT/BCIP Western Detection Chromogenic Kit were provided by Life Technologies (Carlsbad, CA). Osteoassay surface multiwell plates were acquired from Corning Inc. (New York, NY). The bicinchoninic acid (BCA) protein assay kit was purchased from Thermo Scientific (Rockford, IL). Primary rabbit antibodies against phosphorylated forms of Iк $\beta \alpha^{\mathrm{ser} 32 / 36}, \mathrm{p} 38^{\text {thr180/tyr182 }}$ JNK $^{\text {thr183/tyr185 }}$ ERK $^{\text {thr202/tyr204 }}$ and total-c-Fos antibodies were obtained from Signalway Antibody LLC (College Park, MD) and IKK $\alpha / \beta^{\operatorname{ser} 176 / 180}$ and total-NFATc1 antibodies were supplied by Cell Signaling Technology (Beverly, MA). Primary rabbit antibody against GAPDH was obtained from Abcam (Cambridge, MA) and goat anti-rabbit ALP-conjugated secondary antibody was procured from Life Technologies (Carlsbad, CA).

\subsection{Stock Solution}

A $200 \mathrm{mM}$ stock solution of piperine was prepared in DMSO (vehicle) and frozen as aliquots in $-80^{\circ} \mathrm{C}$ until further use. Stock solutions were freshly diluted to working concentrations in complete culture medium before experiments. The final DMSO concentration in the culture medium did not exceed $0.05 \%(\mathrm{v} / \mathrm{v})$.

\subsection{Cell culture}

RAW264.7 murine macrophages (\#TIB-71), MDA-MB-231 (\#HTB-26), MCF-7 (\#HTB-22) human breast cancer cell lines and MC3T3-E1 cells (\#2593) were purchased from American Type Culture Collection (ATCC, Rockville, MD) and maintained in DMEM with $10 \% \mathrm{FBS}$. Cells were incubated at $37^{\circ} \mathrm{C}$ in a humidified atmosphere with $5 \% \mathrm{CO}_{2}$. 


\subsection{Isolation of human CD14+ monocytes and cell culture}

This article does not contain any studies with animals performed by any of the authors. All the procedures and experimental protocols were approved by the Human Research Ethics Committee of the Faculty of Health Sciences, University of Pretoria (Protocol approval number: S154/2012) and in accordance with the 1964 Helsinki declaration and its later amendments. Eligible participants were asked to provide an additional written informed consent for enrolment. Peripheral blood (40-60 ml) was collected from healthy male donors (aged 18-35) into heparinized tubes. Human peripheral blood CD14+ monocytes were isolated as described elsewhere using CD14+ magnetic beads as per manufacturer's instructions (Miltenyi Biotec, San Diego, CA) (26). Cells were cultured in $\alpha$-MEM supplemented with $10 \%$ FBS and incubated at $37^{\circ} \mathrm{C}$ in a humidified atmosphere with $7 \% \mathrm{CO}_{2}$.

\subsection{Alamar blue assay}

Cells were seeded in 96-well plates and after $12 \mathrm{~h}$ of attachment were exposed to increasing concentrations of piperine $(20 \mu \mathrm{M}, 40 \mu \mathrm{M}, 80 \mu \mathrm{M}$ and $100 \mu \mathrm{M})$. Alamar blue assay was conducted as per manufacturer's instructions (Life Technologies). Absorbance was measured at $570 \mathrm{~nm}$ with $600 \mathrm{~nm}$ as reference wavelength on a microplate reader (BioTek Instruments Inc., Winooski, VT).

\subsection{Osteoclast differentiation and TRAP staining}

RAW264.7 macrophages were differentiated into osteoclasts under the influence of RANKL for 5 days as described previously (27). Briefly, cells were suspended in DMEM containing 10\% FBS and seeded into sterile 96-well culture plates at a density of $5 \times 10^{3}$ cells per well. Cells were stimulated with RANKL alone $(15 \mathrm{ng} / \mathrm{ml})$ or in combination with increasing concentrations of piperine $(20 \mu \mathrm{M}, 40 \mu \mathrm{M}, 80 \mu \mathrm{M}$ and $100 \mu \mathrm{M})$. Cell culture media and factors were replaced every third day and differentiation was terminated on the fifth day unless otherwise stated.

Breast cancer cell conditioned medium (CM) was prepared by culturing the cells in T-75 flasks until confluency followed by collection of supernatant and filter sterilization. Resultant $\mathrm{CM}$ was stored as aliquots at $-80^{\circ} \mathrm{C}$ until further use. CM at 10\% was added to the RAW264.7 culture medium and cells were differentiated for 7 days.

CD14+ monocytes were differentiated in the presence of M-CSF (25 ng/ml) and RANKL (30 ng/ml) for 14 days as described previously (26).

Osteoclast specific TRAP staining was performed using a leucocyte acid-phosphatase kit as per manufacturer's directions (Sigma Aldrich, St Louis). TRAP+ cells with 3 or more nuclei were scored as osteoclasts. Photomicrographs were taken with a Zeiss Axiocam MRc5 camera attached to a Zeiss Axiovert 40 CFL microscope (Carl Zeiss AG, Oberkochen, Germany).

\subsection{Pit formation assay}

The bone resorption function of RANKL-induced osteoclasts derived from RAW264.7 cells was analyzed using the osteoassay plates as per manufacturer's instructions (Corning Inc, New York). Resorption pits were observed under a light microscope and quantified by ImageJ software (28). 


\subsection{Actin ring formation assay}

Actin rings of osteoclasts were detected by staining actin filaments with Atto-conjugated phalloidin as described elsewhere (29). Images were acquired using a fluorescence microscope (Carl Zeiss AG, Oberkochen, Germany).

\subsection{Western blot analysis}

Cell lysates were prepared using cell extraction buffer (Life Technologies, Carlsbad), supplemented with protease and phosphatase inhibitors (Sigma Aldrich, St Louis) and resolved on 12\% SDS-PAGE gels. Purified proteins were quantified using a BCA protein assay kit as per manufacturer's directions (Sigma Aldrich, St Louis). Proteins were electrotransferred to nitrocellulose membranes with Tris-glycine transfer buffer [25 mM Tris, $192 \mathrm{mM}$ glycine, 20\% methanol (v/v)] probed with each antibody and detected by NBT/BCIP substrate. Digital images of the blots were acquired using a flatbed scanner (Ricoh Aficio, Johannesburg, South Africa). Band intensities were calculated using ImageJ software (28).

\subsection{Statistical analysis}

Data are representative of three independent experiments unless otherwise stated and are represented as mean \pm standard deviation (SD). Statistical analysis was performed by one-way analysis of variance (ANOVA) followed by Bonferroni post hoc multiple comparison test using Graph Pad Prism Software (GraphPad Software Inc., CA). $P<0.05$ was regarded as statistically significant.

\section{Results}

This study was conducted to analyze the effects of piperine (Fig. 1A) on osteoclast formation induced by breast cancer cells and RANKL, as well as to investigate the effects of piperine on RANKL signaling involved in osteoclastogenesis. RAW264.7 murine macrophages and human CD14+ monocytes (henceforth referred as CD14+ monocytes) were used as osteoclast differentiation models for in vitro and ex vivo studies respectively.

\subsection{Piperine does not affect the cell viability of RAW264.7 macrophages and CD14+ monocytes}

RAW264.7 macrophages and CD14+ monocytes were treated with 20-100 $\mu \mathrm{M}$ of piperine for 48h, $72 \mathrm{~h}$ and $96 \mathrm{~h}$ to analyze cell viability by alamar blue assay. At tested concentrations, piperine did not exert cytotoxic effects on the studied cell lines (Fig. 1B, C). Hence, these concentrations were chosen to study the effects of piperine in downstream experiments.

\subsection{Piperine inhibits RANKL-induced osteoclastogenesis}

RANKL is a major cytokine involved in the induction of osteoclastogenesis. Therefore, we investigated whether piperine can modulate osteoclast differentiation induced by RANKL. The osteoclast progenitor RAW264.7 macrophage cell line was treated with various concentrations $(20-100 \mu \mathrm{M})$ of piperine in the presence or absence of RANKL and allowed to differentiate into osteoclasts for 5 days. As shown in Figure 2A, RANKL-treated cells differentiated into TRAP+ giant multinucleated osteoclasts. However, co-presence of piperine significantly reduced the differentiation of cells into osteoclasts. Furthermore, piperine mediated inhibition of osteoclastogenesis occurred in a dose-dependent manner (Fig. 2B). As low as $80 \mu \mathrm{M}$ piperine had a significant effect on RANKL-induced osteoclastogenesis in RAW264.7 macrophages. 
We next examined whether the anti-osteoclastogenic effects of piperine observed in murine macrophages in vitro could also be interpolated on human cells ex vivo in peripheral blood CD14+ monocytes. Results indicated that RANKL robustly induced osteoclast formation, whereas piperine inhibited this differentiation in a dose-dependent manner in CD14+ monocytes (Fig. 2C, D).

\subsection{Piperine mediated inhibition of RANKL-induced osteoclastogenesis correlates with the onset of osteoclast formation}

To determine the onset of RANKL-induced osteoclast formation and analyze piperine mediated inhibition of osteoclastogenesis we followed the differentiation from days 3, 4 and 5. Stimulation of RAW264.7 macrophages with RANKL induced their differentiation into TRAP+ osteoclasts as early as day 3 as evidenced through morphological observations (Fig. 3A). On the contrary, piperine exposure significantly attenuated RANKLinduced osteoclastogenesis. The extent of suppression was measured by counting the number of TRAP+ osteoclasts per well (Fig. 3B) after days 3, 4, and 5. RANKL-induced osteoclast formation occurred in a timedependent manner with highest number of osteoclasts seen at day 5 (Fig. 3B). However, compared to cells treated with RANKL alone, piperine dose-dependently reduced the number of TRAP+ osteoclasts with maximum inhibition observed at $100 \mu \mathrm{M}$ at all days examined.

\subsection{Piperine inhibits RANKL-induced osteoclastogenesis at an early stage of differentiation}

As seen in earlier experiments, osteoclasts first began to appear from the third day of RANKL exposure in RAW264.7 macrophages and completely differentiated into osteoclasts by the fifth day. To assess whether piperine-mediated inhibition of RANKL-induced osteoclastogenesis is time-dependent, we either treated cells together with RANKL and piperine at day 0 or initially treated the cells with RANKL alone, and piperine was added after days 1, 2, 3, or 4 (Figure 4A, B). As seen in figure 4A, piperine suppressed osteoclast formation when added at day 0 or even after 1 or 2 days of RANKL addition. However, piperine did not affect osteoclast formation when supplemented after days 3 and 4 of RANKL stimulation (Fig.4), indicating inhibitory effects of piperine at early stages of osteoclast differentiation.

\subsection{Piperine inhibits actin ring formation and bone resorption}

Actin ring formation and bone resorption are characterstic hallmarks of osteoclast. Treatment with RANKL induced fusion of RAW264.7 macrophages generating large osteoclasts with actin rings clearly seen as green fluorescence in figure 5A. On the contrary and in line with the osteoclast formation results RAW264.7 macrophages co-treated with piperine $(100 \mu \mathrm{M})$ failed to fuse into osteoclasts and develop actin rings (Fig. 5A). Furthermore, owing to the attenuation of osteoclastogenesis and actin ring formation, RANKL and piperine (100 $\mu \mathrm{M}$ ) co-treated cells did not form resorption pits (Fig. 5B, C). However, RANKL treated cells clearly demonstrated bone resorptive activity (Fig. 5B, C).

\subsection{Piperine inhibits RANKL-induced activation of p38 MAP kinase pathway}

To delineate the molecular mechanisms involved in piperine mediated inhibition of osteoclastogenesis we analysed activation of MAPKs and NF- $\kappa \beta$ pathways at various time-points after RANKL stimulation. Treatment of RAW264.7 macrophages with RANKL led to a steep activation of these pathways as seen by western blot 
through phosphorylation-specific antibodies (Fig. 6). Piperine markedly inhibited p38 activation whereas other signaling pathways including JNK, ERK and NF- $\kappa \beta$ pathways remained unaffected (Fig. 6).

\subsection{Piperine suppresses RANKL-induced c-Fos and NFATc1 expression}

c-Fos and NFATc1 are crucial transcription factors induced at later stages of osteoclast signaling pathway. To analyse the effects of piperine on RANKL-mediated expression of these transcription factors western blotting was performed. As shown in figure 7A and B, RANKL potently induced the expression of c-Fos and NFATc1 as early as $12 \mathrm{~h}$ and $24 \mathrm{~h}$ respectively. However, co-exposure with piperine led to a drastic decrease in the expression levels of c-Fos and NFATc1 (Fig. 7A, B).

\subsection{Piperine inhibits osteoclastogenesis induced by breast cancer cells}

Breast cancer metastasis to bone is associated with osteoclastogenesis and bone loss. To assess whether piperine can modulate breast cancer-induced osteoclastogenesis, we exposed RAW264.7 macrophages to 10\% CM derived from MDA-MB-231 and MCF-7 human breast cancer cells. As shown in Fig. 8A, B incubation of RAW264.7 macrophages with $\mathrm{CM}$ derived from the breast cancer cells led to formation of osteoclasts after 7 days, and piperine suppressed this differentiation.

\subsection{Piperine did not affect the viability of MC3T3-E1 osteoblast-like cells}

Bone remodeling is a closely coupled process involving bone formation and resorption by osteoblasts and osteoclasts respectively. An ideal anti-osteoclastogenic compound should not affect the viability of osteoblasts. To analyze the effects of piperine on the viability of murine MC3T3-E1 osteoblast like cells we further conducted an alamar blue assay. Treatment of MC3T3-E1 cells with piperine (20-100 $\mu \mathrm{M})$ did not affect the viability of these cells (Fig. 9).

\section{Discussion}

Osteoclasts play an important role in bone remodeling by resorbing the old and deteriorating bone. However, during certain conditions such as osteoporosis and cancer metastasis of bone, overactivity of osteoclasts results in uncoupling of the remodeling process leading to excessive osteoclast activity and increased risk of fracture (30). In this study we assessed the effects of piperine on RANKL and breast cancer induced osteoclastogenesis in murine macrophage RAW264.7 cells and human CD14+ monocytes. Our results indicate that piperine inhibits the RANKL and breast cancer-induced osteoclastogenesis by attenuating the RANKL-mediated p38 activation and suppressing the expression of c-Fos and NFATc1 transcription factors. Furthermore, we also show that piperine acts at an early stage of osteoclast differentiation and does not affect the viability of osteoblast-like cells.

Osteoclasts originate from the monocyte/macrophage cells of the hematopoietic lineage (13). Osteoclasts attach to the bone matrix and degrade the mineral and organic components of bone. Since, the last decade considerable information has been accumulated regarding signaling pathways and mechanisms involved in differentiation and activation of osteoclasts (31). RANKL is a major cytokine implicated in osteoclast formation and differentiation. Unregulated RANKL signaling has been reported during various conditions such as ageing, osteoporosis, arthritis and cancer-induced bone loss $(30,5)$. Natural products are a great source of efficacious and affordable lead 
compounds for drug development (16). Piperine, an alkaloid found in Piper nigrum Linn. is such a compound with several bioactive properties (32).

Osteoclast formation is a multi-step process tightly regulated by the MAPKs (p38, JNK and ERK) and NF- $\kappa \beta$ pathways leading to the induction and expression of c-Fos and NFATc1 $(33,8)$. Especially, NFATc1 plays a key role at terminal stages of osteoclast differentiation (34). Abrogation of any of these pathways severely alleviates osteoclast formation and bone resorption (35-37). Our results demonstrate that piperine perturbed the RANKLinduced phosphorylation and activation of p38 MAPKs without affecting the activation of JNK, ERK and NF- $\kappa \beta$ pathways. Earlier published studies have shown that p38-mediated pathway plays an indispensable role in osteoclast formation and pharmacological inhibition of p38 activation severely blunts osteoclastogenesis $(35,38)$. Moreover, p38 has been reported to be crucial for the induction of c-Fos and NFATc1 during RANKL-induced osteoclast formation (39).

c-Fos is a critical transcription factor involved in osteoclastogenesis and induction of downstream genes related with osteoclast differentiation (40). c-Fos knockout mice develop severe osteopetrotic phenotype with complete deficiency of osteoclasts $(41,42)$. In the present study, we found that piperine drastically attenuated the RANKLinduced expression of c-Fos in osteoclast progenitors. It has been reported elsewhere that downregulation of cFos by 1, 25-dihydroxyvitamin D3 results in suppression of osteoclastogenesis (43). Thus c-Fos is a crucial target for inhibiting osteoclastogenesis. NFATc1 is one of the most downstream transcription factors involved in osteoclast formation (34). Expression of NFATc1 has been reported to occur downstream of c-Fos followed by auto-amplification of NFATc1 by its own activity $(44,34)$. NFATc1 knockout mice completely lack osteoclasts and are osteopetrotic (34). Our results in this study demonstrate that piperine negatively regulated the NFATc1 expression. Hence, the anti-osteoclastogenic effects of piperine could be due to its inhibition of p38 signaling pathway that consecutively led to the downregulated expression of c-Fos and NFATc1.

Breast cancer cells are reported to frequently migrate to bone and express proteins that aid in attachment to bone including chemokines (CXCR4) and increase osteolysis via osteoclasts (45). Osteoclastogenesis has also been reported to be triggered by pathways alternative to RANKL such TNF- $\alpha$ induced osteoclastogenic pathway (46). Earlier lines of evidence suggest that breast cancer cell lines employed in this study (MDA-MB-231 and MCF-7) do not express RANKL (47). However, secretion of TNF- $\alpha$ in the conditioned medium of these cell lines has been reported (48). Additionally, Kwak et al. and Zwerina et al., in separate studies reported that p38 signaling pathway plays a critical role downstream of TNF- $\alpha$ in osteoclast differentiation and inflammatory bone loss (49, 50). Inhibition of p38 significantly reduced clinical signs of TNF- $\alpha$-mediated arthritis (50). Our results from this study indicate that piperine inhibited osteoclastogenesis induced by the conditioned-medium derived from the breast cancer MDA-MB-231 and MCF-7 cells. Hence, it is possible that piperine suppressed osteoclast formation induced by breast cancer cells through inhibition of p38 activation.

Anti-resorptive and anabolic therapies are often prescribed in conjunction to patients for increasing bone mass and preventing bone loss. Our results indicate that piperine did not exert cytotoxic effects on osteoblast-like cells supporting its possible efficacy in combination with anabolic agents. Current therapeutic options available for bone loss treatments either involves usage of anti-osteoclastogenic therapies such as bisphosphonates, denosumab or anabolic therapies such as parathyroid hormone (PTH) (51). Long-term usage of bisphosphonates results in 
toxic side effects such as renal impairment and osteonecrosis of the jaw which outweigh the positive effects $(52$, 53). Furthermore, denosumab a humanized monoclonal antibody to RANKL is used as a therapy for patients with post-menopausal osteoporosis and cancer induced bone loss. Usage of this therapy is often implicated with skin problems (eczema, infections), hypocalcemia and osteonecrosis of the jaw (54). Additionally, usage of antibody for therapeutic purposes is costly, unaffordable and often out of reach to many patients. Therefore, cheaper, affordable therapeutic options with minimal or no side effects are much warranted for treating bone loss.

Hence, our study demonstrates that the plant-derived small molecule piperine alleviates osteoclast formation induced by RANKL and breast cancer cells in RAW264.7 macrophages in vitro and by RANKL in human CD14+ monocytes ex vivo. Piperine could be a potential lead compound and a novel source for synthesizing analogues for anti-osteoclastogenic drug development.

\section{Acknowledgments}

The authors declare no competing interests. This study was supported by grants from RESCOM, University of Pretoria; the University of Pretoria Vice Chancellor's Postdoctoral Research Fellowship; the University of Pretoria's Strategic Institutional Research Theme in Food, Nutrition and Well-being; and the StruwigGermeshuysen Research Trust, South Africa.

\section{References}

[1] H. K. Väänänen, T. Laitala-Leinonen (2008) Osteoclast lineage and function. Archives of Biochemistry and Biophysics 473:132-138.

[2] B. Boyce, L. Xing (2007) Biology of RANK, RANKL, and osteoprotegerin. Arthritis Res Ther 9:1-7.

[3] G. Karsenty (2001) Minireview: transcriptional control of osteoblast differentiation. Endocrinology 142:27312733.

[4] K. Vaananen (2005) Mechanism of osteoclast mediated bone resorption--rationale for the design of new therapeutics. Advanced drug delivery reviews 57:959-971.

[5] J. Perez-Garcia, E. Muñoz-Couselo, J. Cortes (2013) Bone metastases: Causes, consequences and therapeutic opportunities. European Journal of Cancer Supplements 11:254-256.

[6] F. Veronesi, P. Torricelli, V. Borsari, M. Tschon, L. Rimondini, M. Fini (2011) Mesenchymal stem cells in the aging and osteoporotic population. Critical reviews in eukaryotic gene expression 21:363-377.

[7] P. L. Chung, S. Zhou, B. Eslami, L. Shen, M. S. LeBoff, J. Glowacki (2014) Effect of age on regulation of human osteoclast differentiation. Journal of cellular biochemistry 115:1412-1419.

[8] A. Leibbrandt, J. M. Penninger (2008) RANK/RANKL: regulators of immune responses and bone physiology. Annals of the New York Academy of Sciences 1143:123-150.

[9] L. C. Hofbauer, A. E. Heufelder, R. G. Erben (2001) Osteoprotegerin, RANK, and RANK ligand: the good, the bad, and the ugly in rheumatoid arthritis. The Journal of rheumatology 28:685-687.

[10] L. Huang, Y. Y. Cheng, L. T. C. Chow, M. H. Zheng, S. M. Kumta (2002) Tumour cells produce receptor activator of NF-kB ligand (RANKL) in skeletal metastases. Journal of Clinical Pathology 55:877-878.

[11] F. Kanamaru, H. Iwai, T. Ikeda, A. Nakajima, I. Ishikawa, M. Azuma (2004) Expression of membrane-bound and soluble receptor activator of NF-kappaB ligand (RANKL) in human T cells. Immunology letters 94:239-246. 
[12] Y. Y. Kong, U. Feige, I. Sarosi, B. Bolon, A. Tafuri, S. Morony, C. Capparelli, J. Li, R. Elliott, S. McCabe, T. Wong, G. Campagnuolo, E. Moran, E. R. Bogoch, G. Van, L. T. Nguyen, P. S. Ohashi, D. L. Lacey, E. Fish, W. J. Boyle, J. M. Penninger (1999) Activated T cells regulate bone loss and joint destruction in adjuvant arthritis through osteoprotegerin ligand. Nature 402:304-309.

[13] Z. Bar-Shavit (2007) The osteoclast: a multinucleated, hematopoietic-origin, bone-resorbing osteoimmune cell. Journal of cellular biochemistry 102:1130-1139.

[14] T. Wada, T. Nakashima, N. Hiroshi, J. M. Penninger (2006) RANKL-RANK signaling in osteoclastogenesis and bone disease. Trends in Molecular Medicine 12:17-25.

[15] A. Leibbrandt, J. M. Penninger (2009) RANK(L) as a key target for controlling bone loss. Advances in experimental medicine and biology 647:130-145.

[16] D. J. Newman, G. M. Cragg (2012) Natural Products as Sources of New Drugs over the 30 Years from 1981 to 2010(). Journal of natural products 75:311-335.

[17] Y. Q. Pei (1983) A review of pharmacology and clinical use of piperine and its derivatives. Epilepsia 24:177182.

[18] R. Mittal, R. Gupta (2000) In vitro antioxidant activity of piperine. Methods Find Exp Clin Pharmacol 22:271274.

[19] L. H. Lai, Q. H. Fu, Y. Liu, K. Jiang, Q. M. Guo, Q. Y. Chen, B. Yan, Q. Q. Wang, J. G. Shen (2012) Piperine suppresses tumor growth and metastasis in vitro and in vivo in a 4T1 murine breast cancer model. Acta pharmacologica Sinica 33:523-530.

[20] P. Shrivastava, K. Vaibhav, R. Tabassum, A. Khan, T. Ishrat, M. M. Khan, A. Ahmad, F. Islam, M. M. Safhi, F. Islam (2013) Anti-apoptotic and Anti-inflammatory effect of Piperine on 6-OHDA induced Parkinson's Rat model. The Journal of Nutritional Biochemistry 24:680-687.

[21] K. Selvendiran, P. Senthilnathan, V. Magesh, D. Sakthisekaran (2004) Modulatory effect of Piperine on mitochondrial antioxidant system in Benzo(a)pyrene-induced experimental lung carcinogenesis. Phytomedicine : international journal of phytotherapy and phytopharmacology 11:85-89.

[22] R. S. Vijayakumar, D. Surya, N. Nalini (2004) Antioxidant efficacy of black pepper (Piper nigrum L.) and piperine in rats with high fat diet induced oxidative stress. Redox report : communications in free radical research 9:105-110.

[23] V. Badmaev, M. Majeed, L. Prakash (2000) Piperine derived from black pepper increases the plasma levels of coenzyme Q10 following oral supplementation. J Nutr Biochem 11:109-113.

[24] M. Kakarala, D. E. Brenner, H. Korkaya, C. Cheng, K. Tazi, C. Ginestier, S. Liu, G. Dontu, M. S. Wicha (2010) Targeting breast stem cells with the cancer preventive compounds curcumin and piperine. Breast cancer research and treatment 122:777-785.

[25] U. H. Park, H. S. Jeong, E. Y. Jo, T. Park, S. K. Yoon, E. J. Kim, J. C. Jeong, S. J. Um (2012) Piperine, a component of black pepper, inhibits adipogenesis by antagonizing PPARgamma activity in 3T3-L1 cells. Journal of agricultural and food chemistry 60:3853-3860.

[26] A. E. Kasonga, V. Deepak, M. C. Kruger, M. Coetzee (2015) Arachidonic acid and docosahexaenoic acid suppress osteoclast formation and activity in human CD14+ monocytes, in vitro. PloS one 10:e0125145. 
[27] V. Deepak, A. Kasonga, M. C. Kruger, M. Coetzee (2015) Inhibitory effects of eugenol on RANKL-induced osteoclast formation via attenuation of NF-kappaB and MAPK pathways. Connective tissue research 56:195-203.

[28] C. A. Schneider, W. S. Rasband, K. W. Eliceiri (2012) NIH Image to ImageJ: 25 years of image analysis. Nature methods 9:671-675.

[29] J. C. Boeyens, V. Deepak, W. H. Chua, M. C. Kruger, A. M. Joubert, M. Coetzee (2014) Effects of omega3and omega6-polyunsaturated fatty acids on RANKL-induced osteoclast differentiation of RAW264.7 cells: a comparative in vitro study. Nutrients 6:2584-2601.

[30] M. H. Helfrich (2003) Osteoclast diseases. Microscopy research and technique 61:514-532.

[31] T. Nakashima, H. Takayanagi (2011) New regulation mechanisms of osteoclast differentiation. Annals of the New York Academy of Sciences 1240:E13-18.

[32] K. Srinivasan (2007) Black pepper and its pungent principle-piperine: a review of diverse physiological effects. Critical reviews in food science and nutrition 47:735-748.

[33] B. K. Hall in: Bones and Cartilage, (2005) (B. K. Hall, ed), Academic Press, San Diego, pp 197-213.

[34] H. Takayanagi, S. Kim, T. Koga, H. Nishina, M. Isshiki, H. Yoshida, A. Saiura, M. Isobe, T. Yokochi, J. Inoue, E. F. Wagner, T. W. Mak, T. Kodama, T. Taniguchi (2002) Induction and activation of the transcription factor NFATc1 (NFAT2) integrate RANKL signaling in terminal differentiation of osteoclasts. Developmental cell 3:889-901.

[35] M. Matsumoto, T. Sudo, T. Saito, H. Osada, M. Tsujimoto (2000) Involvement of p38 mitogen-activated protein kinase signaling pathway in osteoclastogenesis mediated by receptor activator of NF-kappa B ligand (RANKL). The Journal of biological chemistry 275:31155-31161.

[36] J. P. David, K. Sabapathy, O. Hoffmann, M. H. Idarraga, E. F. Wagner (2002) JNK1 modulates osteoclastogenesis through both c-Jun phosphorylation-dependent and -independent mechanisms. Journal of cell science 115:4317-4325.

[37] Y. He, K. Staser, S. D. Rhodes, Y. Liu, X. Wu, S.-J. Park, J. Yuan, X. Yang, X. Li, L. Jiang, S. Chen, F.-C. Yang (2011) Erk1 Positively Regulates Osteoclast Differentiation and Bone Resorptive Activity. PloS one 6:e24780.

[38] X. Li, N. Udagawa, M. Takami, N. Sato, Y. Kobayashi, N. Takahashi (2003) p38 Mitogen-activated protein kinase is crucially involved in osteoclast differentiation but not in cytokine production, phagocytosis, or dendritic cell differentiation of bone marrow macrophages. Endocrinology 144:4999-5005.

[39] H. Huang, E. J. Chang, J. Ryu, Z. H. Lee, Y. Lee, H. H. Kim (2006) Induction of c-Fos and NFATc1 during RANKL-stimulated osteoclast differentiation is mediated by the p38 signaling pathway. Biochemical and biophysical research communications 351:99-105.

[40] B. F. Boyce, T. Yamashita, Z. Yao, Q. Zhang, F. Li, L. Xing (2005) Roles for NF-kappaB and c-Fos in osteoclasts. Journal of bone and mineral metabolism 23 Suppl:11-15.

[41] R. S. Johnson, B. M. Spiegelman, V. Papaioannou (1992) Pleiotropic effects of a null mutation in the c-fos proto-oncogene. Cell 71:577-586.

[42] Z. Q. Wang, C. Ovitt, A. E. Grigoriadis, U. Mohle-Steinlein, U. Ruther, E. F. Wagner (1992) Bone and haematopoietic defects in mice lacking c-fos. Nature 360:741-745. 
[43] H. Takasu, A. Sugita, Y. Uchiyama, N. Katagiri, M. Okazaki, E. Ogata, K. Ikeda (2006) c-Fos protein as a target of anti-osteoclastogenic action of vitamin D, and synthesis of new analogs. The Journal of clinical investigation 116:528-535.

[44] N. Ishida, K. Hayashi, M. Hoshijima, T. Ogawa, S. Koga, Y. Miyatake, M. Kumegawa, T. Kimura, T. Takeya (2002) Large scale gene expression analysis of osteoclastogenesis in vitro and elucidation of NFAT2 as a key regulator. The Journal of biological chemistry 277:41147-41156.

[45] J. J. Yin, C. B. Pollock, K. Kelly (2005) Mechanisms of cancer metastasis to the bone. Cell research 15:5762.

[46] K. Kobayashi, N. Takahashi, E. Jimi, N. Udagawa, M. Takami, S. Kotake, N. Nakagawa, M. Kinosaki, K. Yamaguchi, N. Shima, H. Yasuda, T. Morinaga, K. Higashio, T. J. Martin, T. Suda (2000) Tumor necrosis factor alpha stimulates osteoclast differentiation by a mechanism independent of the ODF/RANKL-RANK interaction. The Journal of experimental medicine 191:275-286.

[47] R. J. Thomas, T. A. Guise, J. J. Yin, J. Elliott, N. J. Horwood, T. J. Martin, M. T. Gillespie (1999) Breast cancer cells interact with osteoblasts to support osteoclast formation. Endocrinology 140:4451-4458.

[48] S. Desai, A. Kumar, S. Laskar, B. N. Pandey (2013) Cytokine profile of conditioned medium from human tumor cell lines after acute and fractionated doses of gamma radiation and its effect on survival of bystander tumor cells. Cytokine 61:54-62.

[49] H. B. Kwak, H. M. Jin, H. Ha, M. J. Kang, S. B. Lee, H. H. Kim, Z. H. Lee (2005) Tumor necrosis factoralpha induces differentiation of human peripheral blood mononuclear cells into osteoclasts through the induction of p21(WAF1/Cip1). Biochemical and biophysical research communications 330:1080-1086.

[50] J. Zwerina, S. Hayer, K. Redlich, K. Bobacz, G. Kollias, J. S. Smolen, G. Schett (2006) Activation of p38 MAPK is a key step in tumor necrosis factor-mediated inflammatory bone destruction. Arthritis and rheumatism 54:463-472.

[51] T. D. Rachner, S. Khosla, L. C. Hofbauer (2011) Osteoporosis: now and the future. Lancet (London, England) 377:1276-1287.

[52] A. Gevorgyan, D. J. Enepekides (2008) Bisphosphonate-induced necrosis of the jaws: a reconstructive nightmare. Current opinion in otolaryngology \& head and neck surgery 16:325-330.

[53] N. B. Watts, D. L. Diab (2010) Long-term use of bisphosphonates in osteoporosis. The Journal of clinical endocrinology and metabolism 95:1555-1565.

[54] D. L. Diab, N. B. Watts (2014) Denosumab in osteoporosis. Expert opinion on drug safety 13:247-253. 


\section{Figure Legends}

Figure 1. Effect of piperine on the viability of RAW264.7 macrophages and CD14+ monocytes. A) Molecular structure of piperine. B) Cell viability of piperine-treated RAW264.7 macrophages (5 x 10\%/well) and C) CD14+ monocytes ( 1 x 10\% /well with $25 \mathrm{ng} / \mathrm{ml} \mathrm{M-CSF})$. Cells were treated with indicated concentrations of piperine for $48 \mathrm{~h}, 72 \mathrm{~h}, 96 \mathrm{~h}$ and cell viability was measured using alamar blue assay. Data are expressed as mean \pm SD percent of control and are representative of three independent experiments performed in triplicate.

Figure 2. Piperine inhibits osteoclastogenesis in RAW264.7 macrophages and CD14+ monocytes. A) RAW 264.7 macrophages $\left(5 \times 10^{3} /\right.$ well) were differentiated with RANKL (15 ng/ml) alone or in combination with piperine at indicated concentrations for 5 days. TRAP+ cells containing three or more nuclei were counted as osteoclasts. Osteoclasts stain purple/pink in the presence of TRAP (scale bars: $50 \mu \mathrm{m}$ ). B) The numbers of TRAP+ multinucleated osteoclasts/well from RAW264.7 macrophages were counted and represented in a graphical format. C) CD14+ monocytes (4 x 104/well) were differentiated with RANKL (15 ng/ml) and M-CSF (25 ng/ml) or in combination with piperine at indicated concentrations for 14 days. D) The number of TRAP+ osteoclasts formed were counted and represented in a graphical format (scale bars: $50 \mu \mathrm{m}$ ). The results are mean $\pm \mathrm{SD}$ and are representative of three independent experiments performed in triplicate. $(* P<0.05, * * P<0.01, * * * P<0.001$ versus RANKL).

Figure 3. Piperine attenuates osteoclastogenesis from the onset of the differentiation. A) RAW 264.7 macrophages $\left(5 \times 10^{3} /\right.$ well) were differentiated with RANKL $(15 \mathrm{ng} / \mathrm{ml})$ alone or in combination with piperine $(100 \mu \mathrm{M})$ for 3, 4 or 5 days and stained for TRAP (scale bars: $50 \mu \mathrm{m}$ ). B) Quantification of multinucleated osteoclasts (containing three or more nuclei) after treatment with RANKL alone or RANKL plus indicated concentrations of piperine for 3, 4, or 5 days. Values represent mean $\pm \mathrm{SD}$ and are representative of three independent experiments performed in triplicate. $(* P<0.05, * * P<0.01, * * * P<0.001$ versus RANKL).

Figure 4. Piperine inhibits osteoclastogenesis at an early stage of differentiation. A) RAW 264.7 macrophages $\left(5 \times 10^{3} /\right.$ well) were differentiated with RANKL ( $\left.15 \mathrm{ng} / \mathrm{ml}\right)$ alone (topmost panel) or in combination with piperine $(100 \mu \mathrm{M})$ as indicated and stained for TRAP on the fifth day of differentiation. B) Multinucleated osteoclasts containing three or more nuclei were counted (scale bars: $50 \mu \mathrm{m}$ ). Values represent mean $\pm \mathrm{SD}$ and are representative of three independent experiments performed in triplicate. ( $*<0.05$, $* * * P<0.001$ versus RANKL).

Figure 5. Piperine inhibits actin ring formation and bone resorption. RAW264.7 macrophages were differentiated into osteoclasts in the presence of RANKL alone or with piperine $(100 \mu \mathrm{M})$ and, A) were stained for actin ring formation with phalloidin (scale bars: $10 \mu \mathrm{m}$ ) or B) were tested for resorption pit formation on osteoassay plates (scale bars: $50 \mu \mathrm{m}$ ). C) Resorption pit formation was quantified with ImageJ software. Arrows represent resorbed area. Results are representative of two independent experiments.

Figure 6. Piperine suppresses the phosphorylation and activation of p38. RAW264.7 macrophages were pretreated with or without piperine $(1000 \mathrm{M})$ for $4 \mathrm{~h}$ prior to RANKL $(35 \mathrm{ng} / \mathrm{ml})$ stimulation at the indicated time 
points. Cell lysates were analyzed by western blot analysis with indicated antibodies. Results are representative of three independent experiments. Band intensities were calculated using ImageJ software.

Figure 7. Piperine suppresses RANKL-induced c-Fos and NFATc1 expression. A,B) RAW264.7 macrophages were cultured in the presence of RANKL alone or with piperine $(100 \mu \mathrm{M})$ for the indicated time periods and whole cell lysates were subjected to western blot analysis with indicated antibodies. Results are representative of two independent experiments. Band intensities were calculated using ImageJ software.

Figure 8. Piperine suppresses osteoclastogenesis induced by breast cancer cells. RAW 264.7 macrophages (5 x 103/well) were differentiated for 7 days in the presence of $10 \%$ CM from A) MCF-7 or B) MDA-MB-231 breast cancer cells alone or in the co-presence of piperine $(100 \mu \mathrm{M})$. Resultant TRAP+ osteoclasts containing three or more nuclei were counted (scale bars: $50 \mu \mathrm{m}$ ). Values represent mean $\pm \mathrm{SD}$ and are representative of three independent experiments performed in triplicate. $(* * * P<0.001$ versus $C M)$.

Figure 9. Effect of piperine on the viability of MC3T3-E1 cells. Cells were treated with the indicated concentrations of piperine for $48 \mathrm{~h}$, cell viability was measured using alamar blue assay. Data are expressed as mean $\pm \mathrm{SD}$ percent of control and are representative of three independent experiments performed in triplicate. 


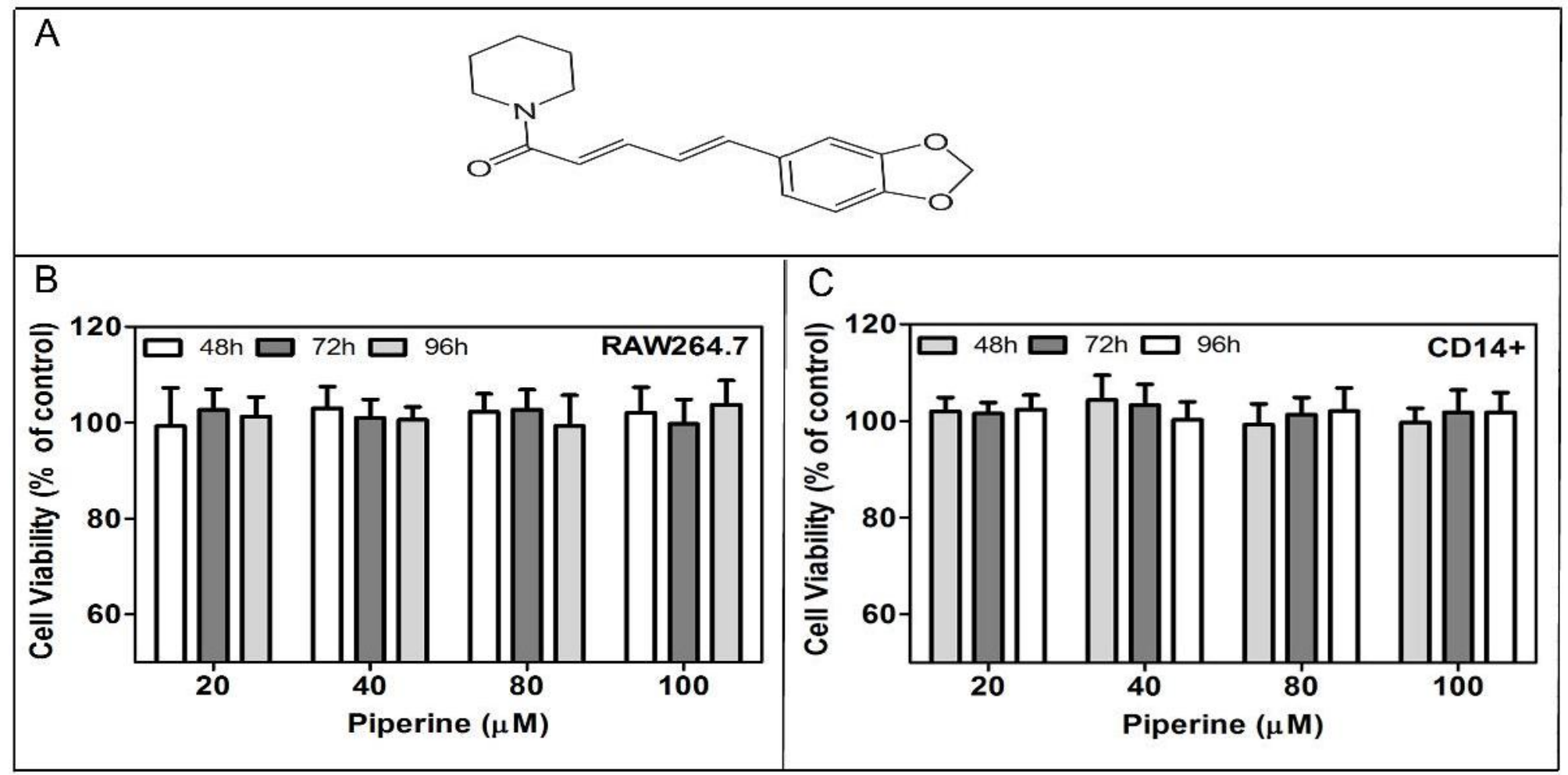




\section{A}
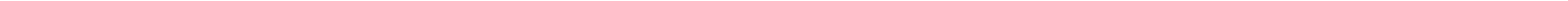

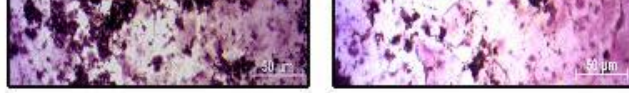

Piperine $(\mu \mathrm{M})$
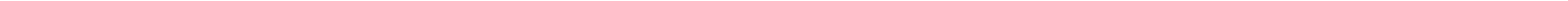

and $x^{2} \mathrm{x}^{2}$
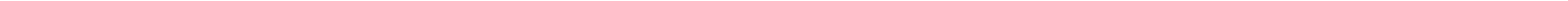

(x)
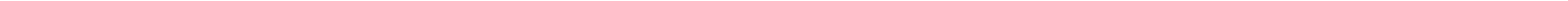

C
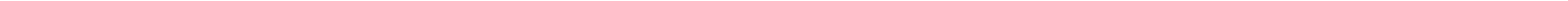

20

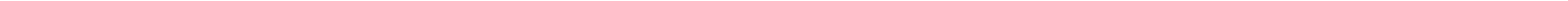

40
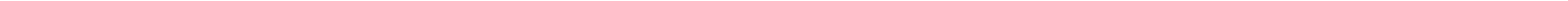

80

100

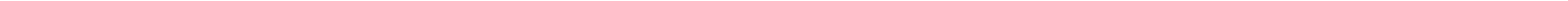

Piperine $(\mu \mathrm{M})$

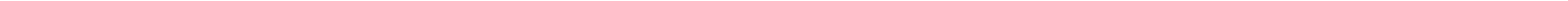

$$
\text { Piperine }(\mu \mathrm{M})
$$




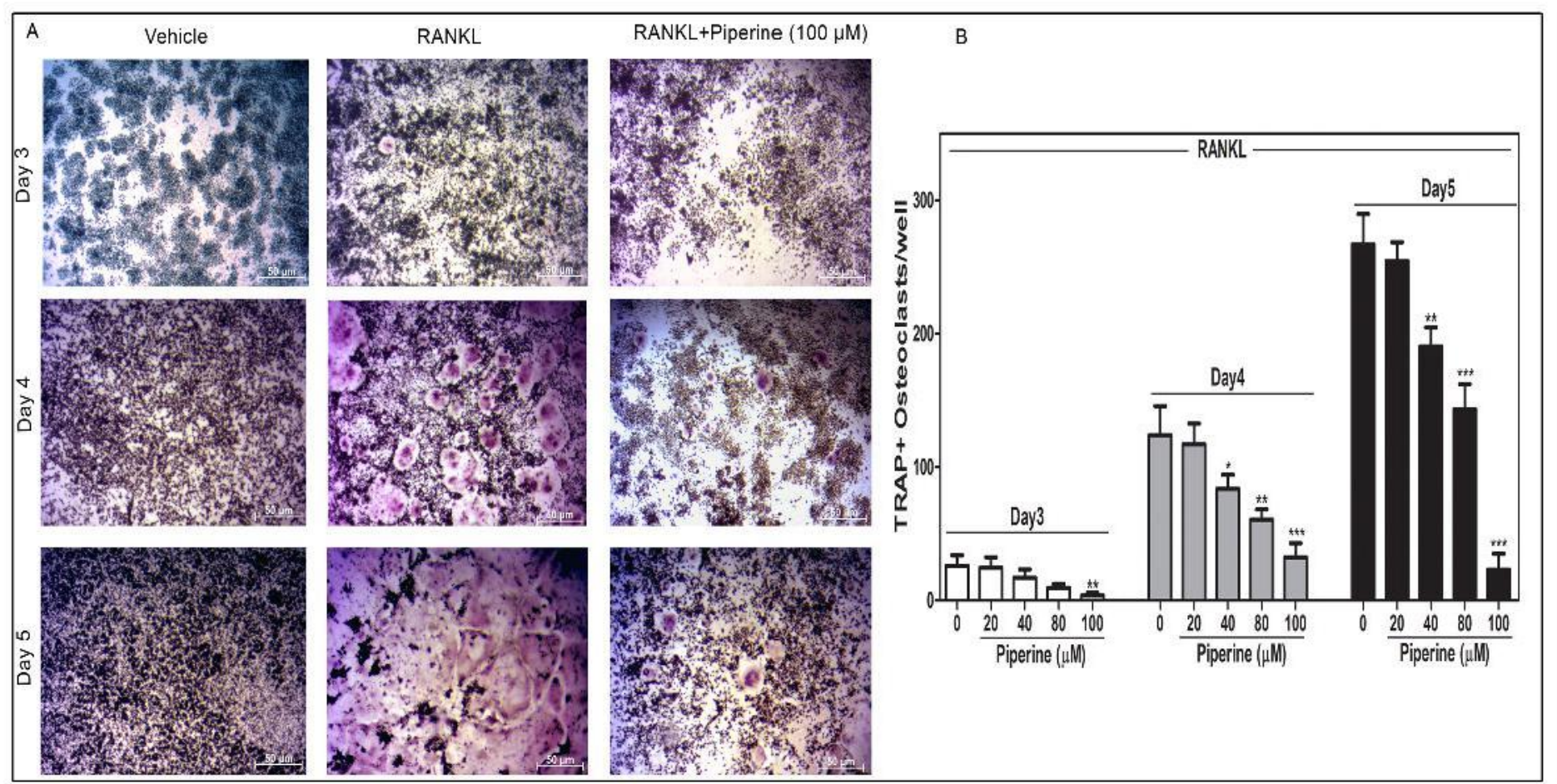




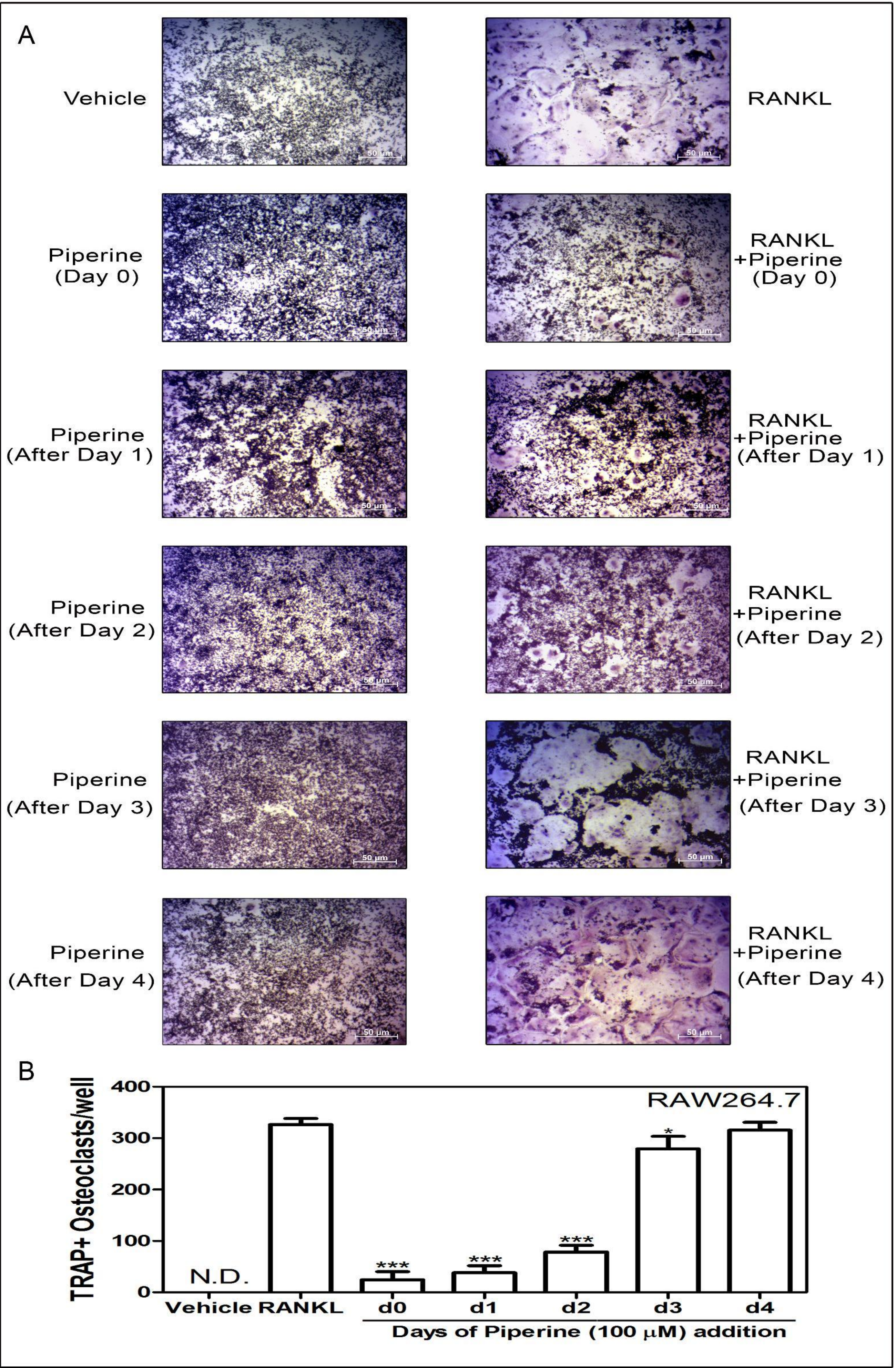



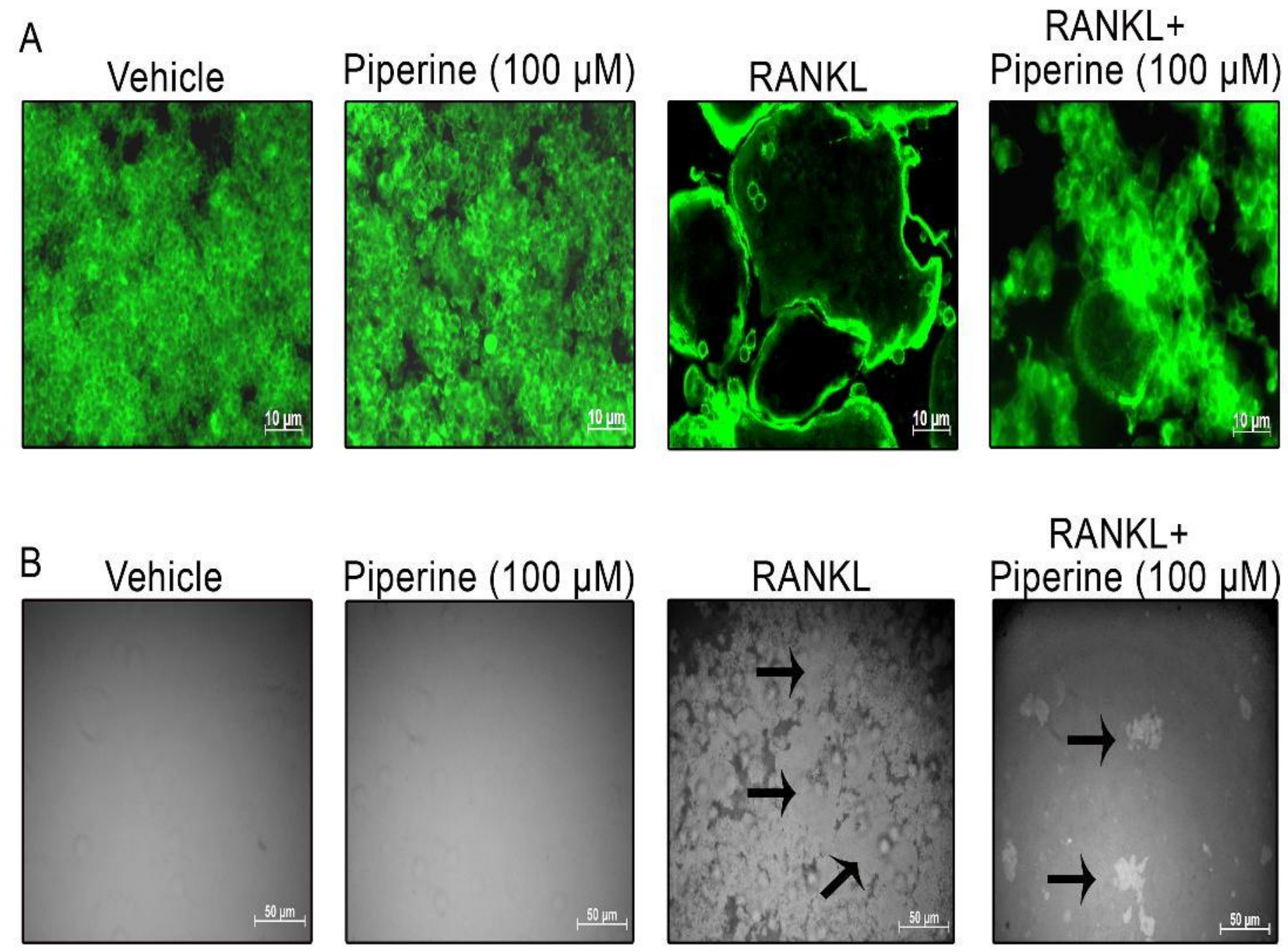

C

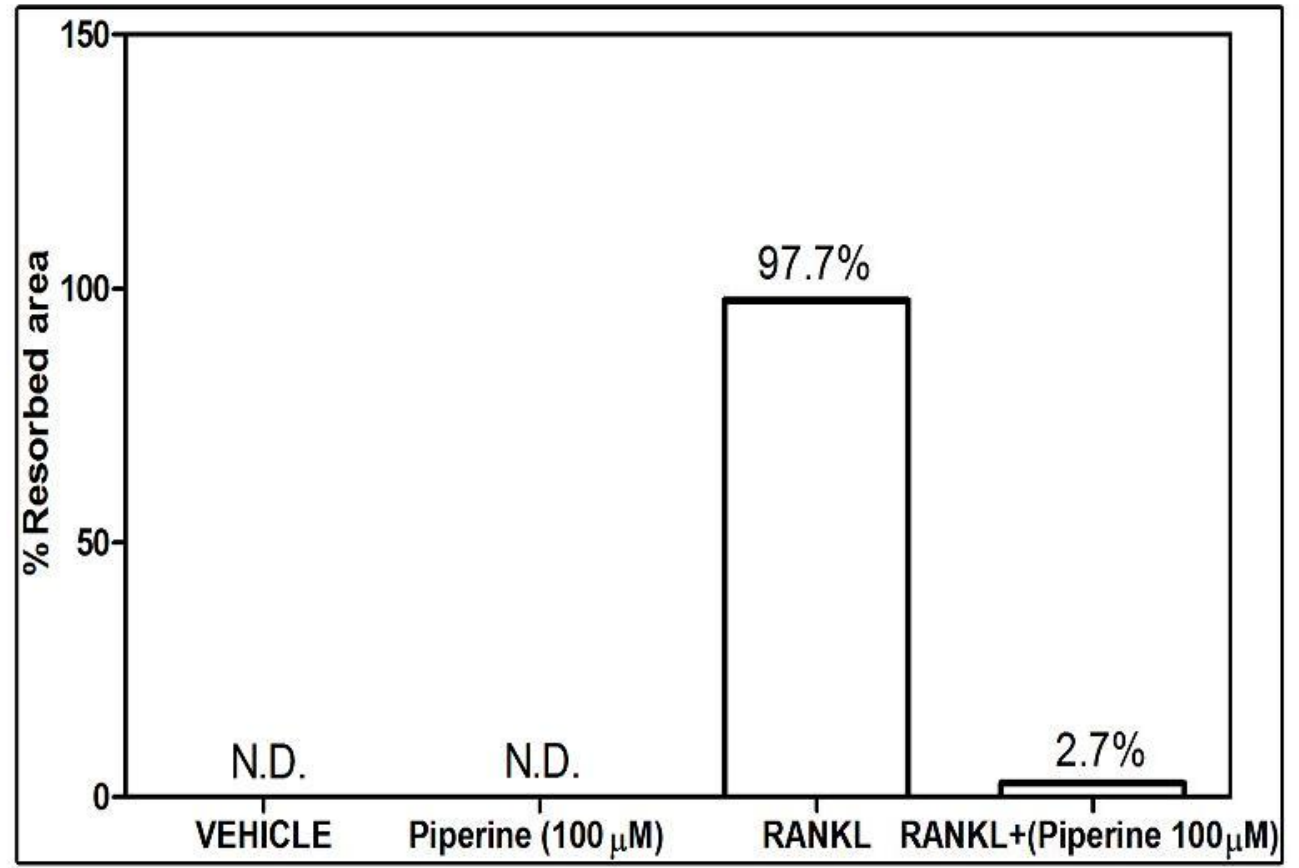




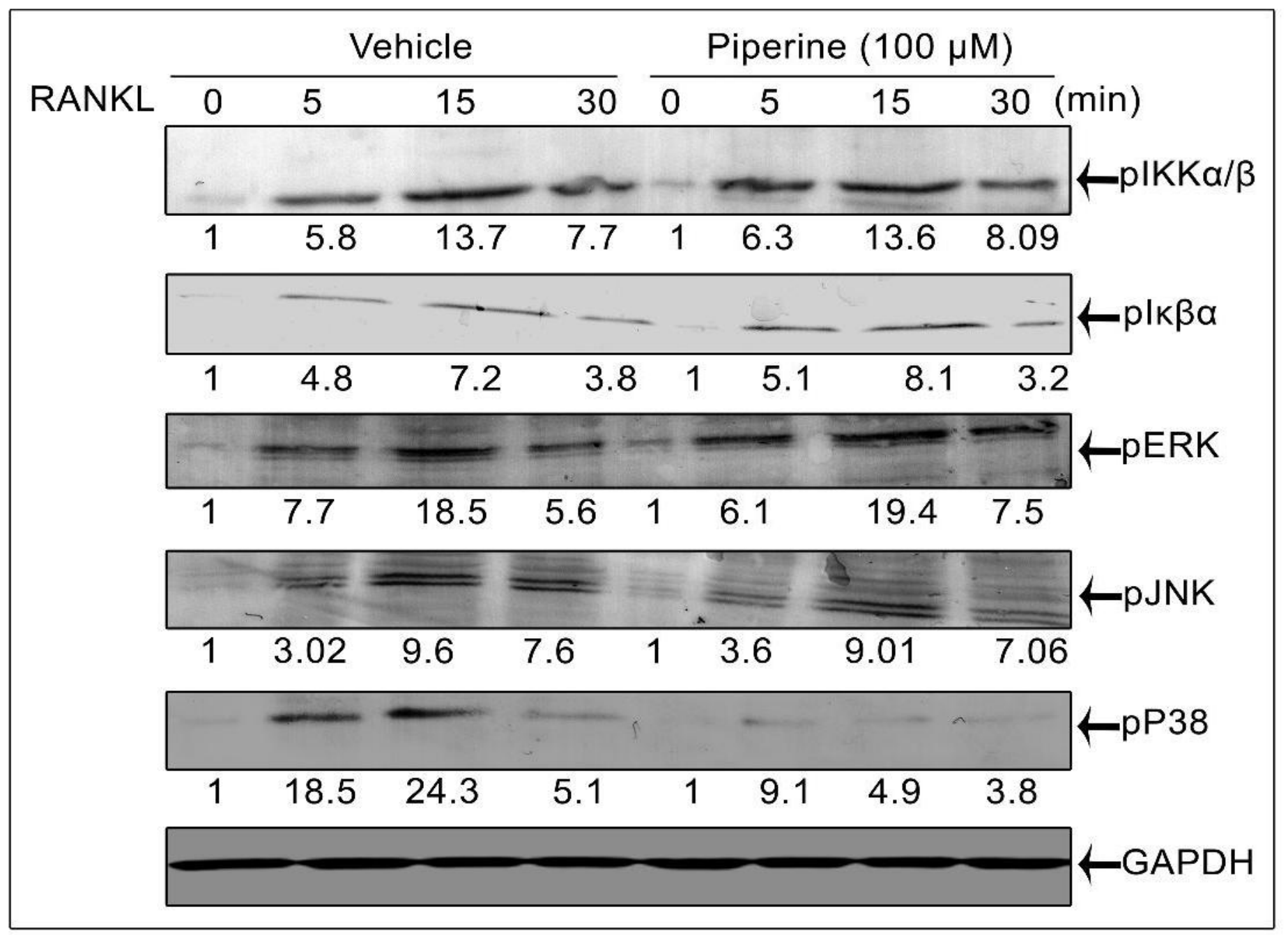




\begin{tabular}{|c|c|c|c|c|c|c|c|}
\hline \multirow{2}{*}{$\begin{array}{l}\text { A } \\
\text { RANKL }\end{array}$} & \multicolumn{3}{|c|}{ Vehicle } & \multicolumn{4}{|c|}{ Piperine $(100 \mu \mathrm{M})$} \\
\hline & 12 & 24 & 48 & 0 & 12 & $24 \quad 48$ & (h) \\
\hline- & $=$ & $=$ & $=$ & $\ldots$ & - & $-\infty=$ & $\vdash$ c-Fos \\
\hline 1 & 5 & 16.1 & 10 & 1 & 2.6 & 4.7 & \\
\hline
\end{tabular}

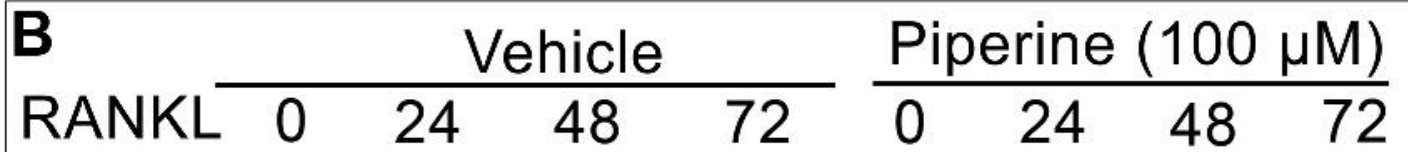
\begin{tabular}{l} 
Piperine $(100 \mu \mathrm{M})$ \\
\hline $0 \quad 24 \quad 48 \quad 72(\mathrm{~h})$
\end{tabular}

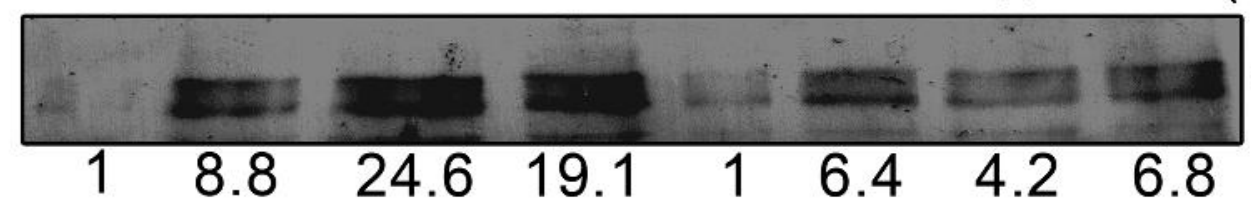
$\leftarrow$ NFATc1 $\begin{array}{llllllll}1 & 8.8 & 24.6 & 19.1 & 1 & 6.4 & 4.2 & 6.8\end{array}$ $\leftarrow$ GAPDH 

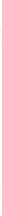

B

Vehicle

+MDA-MB-231 (10\% CM)
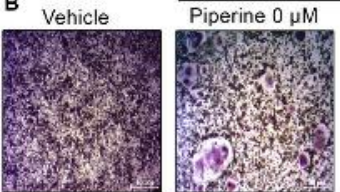

Piperine $100 \mu \mathrm{M}$
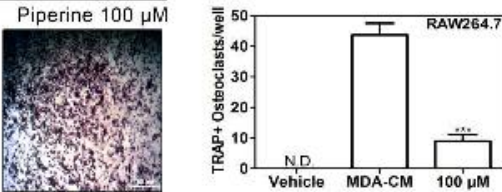


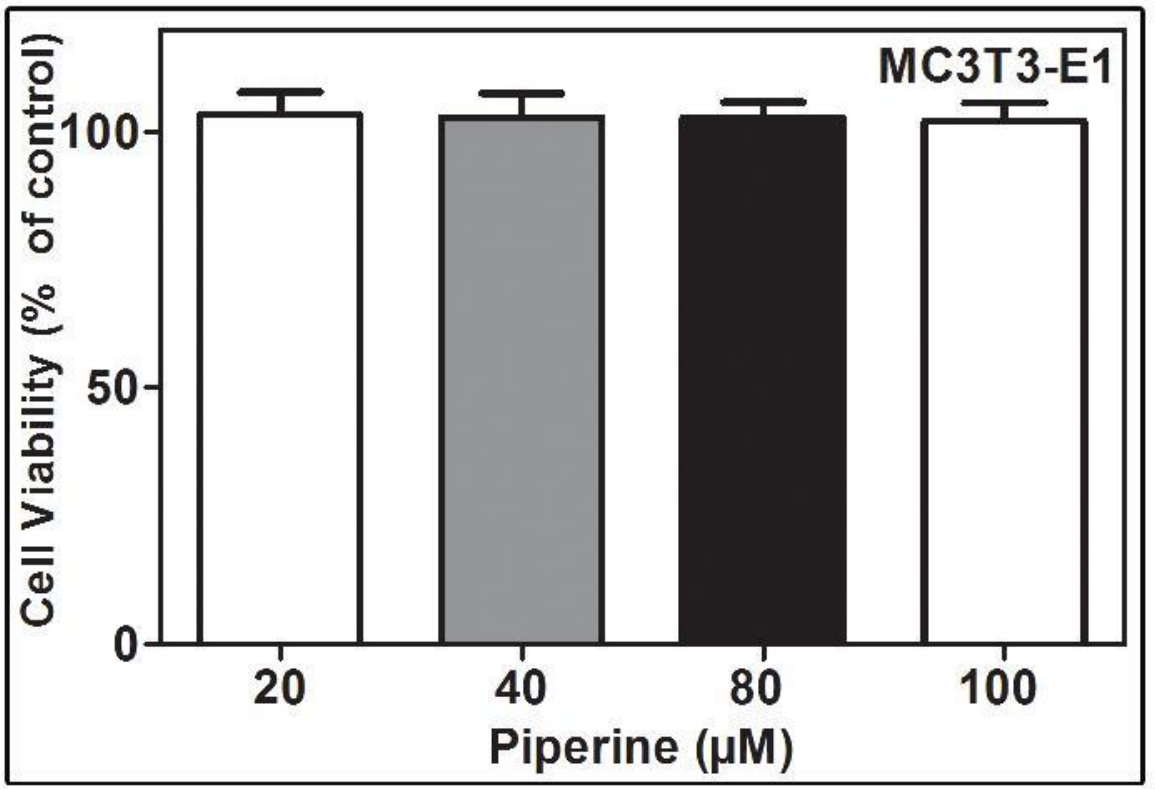

